Article

\title{
Design of a Segmented Mirror with a Global Radius of Curvature Actuation System: Contributions of Multiple Surrogates
}

\author{
Songhang $\mathrm{Wu}^{1,2, *}$, Jihong Dong ${ }^{1, *}$, Shuyan $\mathrm{Xu}{ }^{1}$, Zhirong $\mathrm{Lu}{ }^{1,2}$ and Boqian $\mathrm{Xu}^{1}$ \\ 1 Changchun Institute of Optics, Fine Mechanics and Physics, Chinese Academy of Sciences, \\ Changchun 130033, China; xusy@ciomp.ac.cn (S.X.); luzhirong18@mails.ucas.edu.cn (Z.L.); \\ xuboqian@ciomp.ac.cn (B.X.) \\ 2 University of Chinese Academy of Sciences, Beijing 100049, China \\ * Correspondence: wusonghang17@mails.ucas.ac.cn (S.W.); dongjihong2002@sohu.com (J.D.)
}

Received: 2 November 2020; Accepted: 21 November 2020; Published: 25 November 2020

\begin{abstract}
Due to fabrication difficulties, separately-polished segmented mirrors cannot meet the co-phasing surface shape error requirements in the segmented telescope system. Applying the global radius of curvature (GRoC) actuation system for the individual segments has become an effective solution in space-based telescopes. In this paper, we designed a segmented mirror with a GRoC actuation system. The direct optimization by numerical simulations has low computational efficiency and is not easy to converge for optimizing the actuation point's position on the segmented mirror. For this problem, three common surrogates, including polynomial response surface (PRS), radial basis function neural network (RBFNN), and kriging (KRG), were summed to propose the multiple surrogates (MS) which have the higher approximate ability. The surrogates were then optimized through the multi-island genetic algorithm (MIGA), and the segmented mirror met the design requirement. Compared with direct optimization through numerical simulations, the results show that the proposed multiple-surrogate-based optimization (MSBO) methodology saves computational cost significantly. Besides, it can be deployed to solve other complex optimization problems.
\end{abstract}

Keywords: multiple-surrogate-based optimization; multi-island genetic algorithm; Latin hypercube sampling; segmented mirror with a GRoC actuation system

\section{Introduction}

There exist significant interest in using segmented mirrors instead of a monolithic primary mirror to build a large-aperture telescope. The use of segmented mirrors reduces the difficulties in fabrication, transportation, and replication. Meanwhile, one challenge in the approach lies in matching the individual mirrors to yield a continuous surface. Although the six degrees of freedom adjustments in the segmented-mirror-matching process can correct some aberrations, it cannot fix the surface shape error [1]. Therefore, the existing segmented telescope system applies an active-surface shape-adjustment method. For the ground telescope, a semi-active warping harness structure was adopted in the Keck Telescope. Then, the $30 \mathrm{~m}$ telescope (TMT) and the European Very Large Telescope (E-ELT) also adopted this scheme [2,3]. For the space telescope, taking the James Webb Space Telescope (JWST) as an example, a global radius of curvature (GRoC) actuation system was designed for the non-insistency in the radius of curvature of segmented mirrors. This system has two main applications: (1) After rough polishing of the JWST primary mirror, use of the GRoC actuation system to adjust the GRoC to meet the requirements and record the surface shape error at this time. After removing the GRoC actuation system and supporting structures, the registered surface shape error is used as a 
target map for the finely-polished. The surface shape error root mean square (RMS) after the second polishing can reach $20 \mathrm{~nm}[4,5]$. (2) In the co-phasing process, the system performs micro-control on the GRoC. The first application has a broad adjustment range $( \pm 5 \mathrm{~mm})$, and the second application has a small adjustment range $( \pm 0.15 \mathrm{~mm})$ [6]. Since the actuation point of the GRoC actuation system on the segmented mirror's back directly affects the curvature adjustment accuracy, the actuation point's position needs to be optimized.

Complicated structural optimization through numerical simulations is unavailable since searching for the optimum typically requires thousands or even millions of simulations. To alleviate the computational burden, the surrogate-based optimization methodology (SBOM) becomes the right choice. It simplifies the relationship between output responses and design variables through surrogates, then directly optimizes the surrogates. Wang et al. optimized the $2.8 \mathrm{~m}$ diameter circular mirror's main structural parameters based on the back propagation (BP) neural network surrogate. The error between the surrogate and the simulation was less than 7.09\% [7]. Hong et al. and Xiang et al. used the radial basis function neural network (RBFNN) for the design of the sealing structure and shelter location problem under uncertainty of road networks, respectively, which significantly improved optimization efficiency [8,9]. Ribeiro et al. optimized the functionally-graded plates using an RBFNN surrogate [10]. Wang et al. carried out a many-objective optimization for a deep-sea aquaculture vessel by RBFNN surrogate [11]. Based on the response surface method (RSM) surrogate, J.C. Hsiao et al. carried out a multi-objective optimization design on the robot arm's size parameters, which improved the computational efficiency by $90 \%$ compared with optimization by numerical simulation [12]. Ahmed et al. used an RSM surrogate to replace the 3-D finite element method models in the design of a transverse-flux permanent magnet linear synchronous motor [13]. With the help of an RSM surrogate, Rafiee et al. and Chau et al. achieved good results in designing an outer rotor permanent magnet motor and designing a new compliant planar spring, respectively $[14,15]$. Hakjin et al. used a Kriging surrogate to replace the complicated optimization problem of multiple wing sails considering the flow interaction effect, which effectively improved the computational efficiency [16]. Zhenxu et al. built a Kriging surrogate based on the aerodynamic noise's influence on a high-speed trains's shape design and performed multi-objective optimization [17]. Guo et al. and Das et al. optimized the stiffness of composite cylinders and the nonlinear energy sink by Kriging surrogate, respectively, and the optimization efficiency was remarkably improved $[18,19]$.

However, the approximate ability of the surrogate is insufficient for some complicated optimization, mainly in: (1) the approximate ability of different surrogates is different, and it is not easy to find the best surrogate for one optimization; and (2) a surrogate is easily affected by changes in sample points, and it is difficult to maintain the approximation ability as the number of sample points increases.

To solve this problem, we proposed multiple surrogates to optimize the activation point's position on the segmented mirror's back. The multiple surrogates aimed to minimize the generalized mean square error (GMSE) of the output response and select the combination that was closest to the output response among surrogates (RBFNN [9], PRS [20], and KRG [21]). After the MIGA optimized them, the segmented mirror had met the design requirement. This work attempts to provide an efficient methodology to solve the complicated optimization problem in the design of a segmented mirror with a GRoC actuation system.

The rest of this paper is organized as follows: In Section 2, the multiple surrogates and the multiple-surrogate-based optimization methodology are described and a method to solve the superposition coefficients is introduced. The design process of a segmented mirror with a GRoC actuation system, including the support design and the actuation point's position design, is described in Section 3. Finally, conclusions are drawn in Section 4. 


\section{Proposed Multiple-Surrogate-Based Optimization Methodology}

\subsection{Multiple Surrogates}

The surrogate is a mathematical method to predict the unknown point's responses by building an approximate relationship between design variables and responses relying on limited sample point data information. Several expressions of popular surrogate models, including PRS, RBFNN, and KRG, are as follows:

$$
\text { PRS : } y_{\mathrm{p}}=a_{0}+\sum_{i=1}^{N} b_{i} x_{i}+\sum_{i=1}^{N} c_{i i} x_{i}{ }^{2}+\sum_{i=1}^{N-1} \sum_{j=i+1}^{N} d_{i j} x_{i} x_{j},
$$

where $y_{\mathrm{p}}$ is the output of PRS, $x_{i}$ and $x_{j}$ are the input variables, $N$ is the number of input variables, and $a_{0}, b_{i}, c_{i i}$, and $d_{i j}$ are regression coefficients.

$$
\operatorname{RBFNN}: y_{\mathrm{r}}=\sum_{i=1}^{M} \alpha_{i} \varphi\left(\left\|x-x_{i}\right\|, c\right),
$$

where $y_{\mathrm{r}}$ is the output of RBFNN, $x$ is the input variable, $M$ is the number of input variables, $\alpha_{i}$ is coefficient, || $\|$ is the Euler norm, $\varphi()$ is basis function, and spread constant, $c$, is 0.5 .

$$
\mathrm{KRG}: y_{\mathrm{k}}=\sum_{i=1}^{K} \beta_{i} \phi_{i}(x)+z(x)
$$

where $y_{\mathrm{k}}$ is the output of KRG, $x$ is the input variable, $K$ is the number of input variables, $\beta_{i}$ is coefficient, $\phi_{i}()$ is basis function, and $z()$ is the function of random error.

The approximation accuracy of a surrogate is affected by sampling points. In other words, the best surrogate may be the worst one in the next iteration. And multiple surrogates are linearly superimposed by surrogate models. The best combination of surrogates for approximation can be gained by solving the coefficients during iterations, as follows:

$$
y(x)=w_{\mathrm{p}} y_{\mathrm{p}}(x)+w_{\mathrm{r}} y_{\mathrm{r}}(x)+w_{\mathrm{k}} y_{\mathrm{k}}(x),
$$

where $x$ is the design variables, $y(x)$ is the output of multiple surrogate model, $y_{\mathrm{p}}(x), y_{\mathrm{r}}(x)$, and $y_{\mathrm{k}}(x)$, respectively, are the output of PRS, RBFNN, and KRG, and $w_{\mathrm{p}}, w_{\mathrm{r}}$, and $w_{\mathrm{k}}$ are coefficients. The key to multiple surrogates is to solve coefficients $w_{\mathrm{p}}, w_{\mathrm{r}}$, and $w_{\mathrm{k}}$. Viana et al. suggested that generalized mean square error (GMSE) is suitable for filtering out inaccurate surrogates [22], and it is as follows:

$$
\text { GMSE }=\frac{1}{n} \sum_{i=1}^{n}\left(f_{i}-f_{\mathrm{a}}\right)^{2},
$$

where $n$ is the number of sample points, and $f_{i}$ and $f_{\mathrm{a}}$ represent the prediction output and actual output at $x_{i}$, respectively. Using the GMSE to identify the approximation accuracy of the multiple surrogates, means that it is smaller, and the outputs of the multiple surrogates are closer to the real responses. Therefore, solving coefficients in Equation (4) can be converted into an optimization problem aimed at the smallest GRMS. The optimization problem is as follows:

$$
\left\{\begin{array}{c}
\text { Find }: w_{\mathrm{p}}, w_{\mathrm{r}}, w_{\mathrm{k}} \\
\text { Min }: G=\frac{1}{L} \sum_{i=1}^{L}\left[y\left(x_{i}\right)-y_{\mathrm{a}}\left(x_{i}\right)\right]^{2}
\end{array}\right.
$$


where $G$ is the GMSE of multiple surrogates, $y\left(x_{i}\right)$ is the prediction output at $x_{i}$ using the multiple surrogate models, $y_{\mathrm{a}}\left(x_{i}\right)$ is the actual output at $x_{i}$, and $L$ is the number of sampling points. To find the partial derivatives of $G$ with respect to $w_{\mathrm{p}}$, $w_{\mathrm{r}}$, and $w_{\mathrm{k}}$, respectively,

$$
\begin{aligned}
\frac{\partial G}{\partial w_{\mathrm{p}}} & =\frac{2}{L}\left(w_{\mathrm{p}} \sum_{i=1}^{L} y_{\mathrm{p}}{ }^{2}\left(x_{i}\right)+w_{\mathrm{r}} \sum_{i=1}^{L} y_{\mathrm{r}}\left(x_{i}\right) \sum_{i=1}^{L} y_{\mathrm{p}}\left(x_{i}\right)+w_{\mathrm{k}} \sum_{i=1}^{L} y_{\mathrm{k}}\left(x_{i}\right) \sum_{i=1}^{L} y_{\mathrm{p}}\left(x_{i}\right)-\sum_{i=1}^{L} y_{\mathrm{p}}\left(x_{i}\right) \sum_{i=1}^{L} y_{\mathrm{a}}\left(x_{i}\right)\right), \\
\frac{\partial G}{\partial w_{\mathrm{r}}} & =\frac{2}{L}\left(w_{\mathrm{p}} \sum_{i=1}^{L} y_{\mathrm{p}}\left(x_{i}\right) \sum_{i=1}^{L} y_{\mathrm{r}}\left(x_{i}\right)+w_{\mathrm{r}} \sum_{i=1}^{L} y_{\mathrm{r}}^{2}\left(x_{i}\right)+w_{\mathrm{k}} \sum_{i=1}^{L} y_{\mathrm{k}}\left(x_{i}\right) \sum_{i=1}^{L} y_{r}\left(x_{i}\right)-\sum_{i=1}^{L} y_{r}\left(x_{i}\right) \sum_{i=1}^{L} y_{\mathrm{a}}\left(x_{i}\right)\right), \\
\frac{\partial G}{\partial w_{\mathrm{k}}} & =\frac{2}{L}\left(w_{\mathrm{p}} \sum_{i=1}^{L} y_{\mathrm{p}}\left(x_{i}\right) \sum_{i=1}^{L} y_{k}\left(x_{i}\right)+w_{\mathrm{r}} \sum_{i=1}^{L} y_{\mathrm{r}}\left(x_{i}\right) \sum_{i=1}^{L} y_{\mathrm{k}}\left(x_{i}\right)+w_{\mathrm{k}} \sum_{i=1}^{L} y_{\mathrm{k}}^{2}\left(x_{i}\right)-\sum_{i=1}^{L} y_{k}\left(x_{i}\right) \sum_{i=1}^{L} y_{a}\left(x_{i}\right)\right),
\end{aligned}
$$

By setting the partial derivatives to 0 , the coefficients, $w=\left[\begin{array}{lll}w_{\mathrm{p}} & w_{\mathrm{r}} & w_{\mathrm{k}}\end{array}\right]^{T}$, is solved as follows:

$$
\begin{aligned}
& w=A^{-1} B, \\
& B=\left[\sum_{i=1}^{L} y_{\mathrm{p}}\left(x_{i}\right) \sum_{i=1}^{L} y_{\mathrm{a}}\left(x_{i}\right) \sum_{i=1}^{L} y_{r}\left(x_{i}\right) \sum_{i=1}^{L} y_{\mathrm{p}}\left(x_{i}\right) \quad \sum_{i=1}^{L} y_{\mathrm{k}}\left(x_{i}\right) \sum_{i=1}^{L} y_{\mathrm{p}}\left(x_{i}\right)\right]^{T}, \\
& A=\left[\begin{array}{ccc}
\sum_{i=1}^{L} y_{\mathrm{p}}^{2}\left(x_{i}\right) & \sum_{i=1}^{L} y_{\mathrm{r}}\left(x_{i}\right) \sum_{i=1}^{L} y_{\mathrm{p}}\left(x_{i}\right) & \sum_{i=1}^{L} y_{\mathrm{k}}\left(x_{i}\right) \sum_{i=1}^{L} y_{\mathrm{p}}\left(x_{i}\right) \\
\sum_{i=1}^{L} y_{\mathrm{p}}\left(x_{i}\right) \sum_{i=1}^{L} y_{\mathrm{r}}\left(x_{i}\right) & \sum_{i=1}^{L} y_{\mathrm{r}}^{2}\left(x_{i}\right) & \sum_{i=1}^{L} y_{\mathrm{k}}\left(x_{i}\right) \sum_{i=1}^{L} y_{\mathrm{r}}\left(x_{i}\right) \\
\sum_{i=1}^{L} y_{\mathrm{p}}\left(x_{i}\right) \sum_{i=1}^{L} y_{\mathrm{k}}\left(x_{i}\right) & \sum_{i=1}^{L} y_{\mathrm{r}}\left(x_{i}\right) \sum_{i=1}^{L} y_{\mathrm{k}}\left(x_{i}\right) & \sum_{i=1}^{L} y_{\mathrm{k}}^{2}\left(x_{i}\right)
\end{array}\right],
\end{aligned}
$$

After the multiple surrogates are built, it is necessary to estimate approximation accuracy. Generally, the determination coefficient, $R^{2}$, is a measure used to assess how well a model can explain and predict outcomes. It ranges from 0 to 1 and will be closer to 1 when the model is more precise. In this work, the multiple surrogates with $R^{2}$ greater than 0.9 can replace the simulation model. $R^{2}$ can be obtained as follows:

$$
R^{2}=1-\frac{\sum_{k=1}^{p}\left(y_{\mathrm{r}}\left(x_{k}\right)-y_{a}\left(x_{k}\right)\right)^{2}}{\sum_{k=1}^{p}\left(y_{\mathrm{r}}\left(x_{k}\right)-\bar{y}\right)^{2}},
$$

where $y_{r}\left(x_{k}\right)$ and $y_{a}\left(x_{k}\right)$ are the output of multiple surrogates and the actual model at $x_{k}$, respectively, $p$ is the number of test points, and $\bar{y}$ is the average of the actual output.

\subsection{Procedure of Multiple-Surrogate-Based Optimization}

A stepwise procedure of the proposed multiple-surrogate-based optimization methodology is explained as follows, also with the help of Figure 1. 


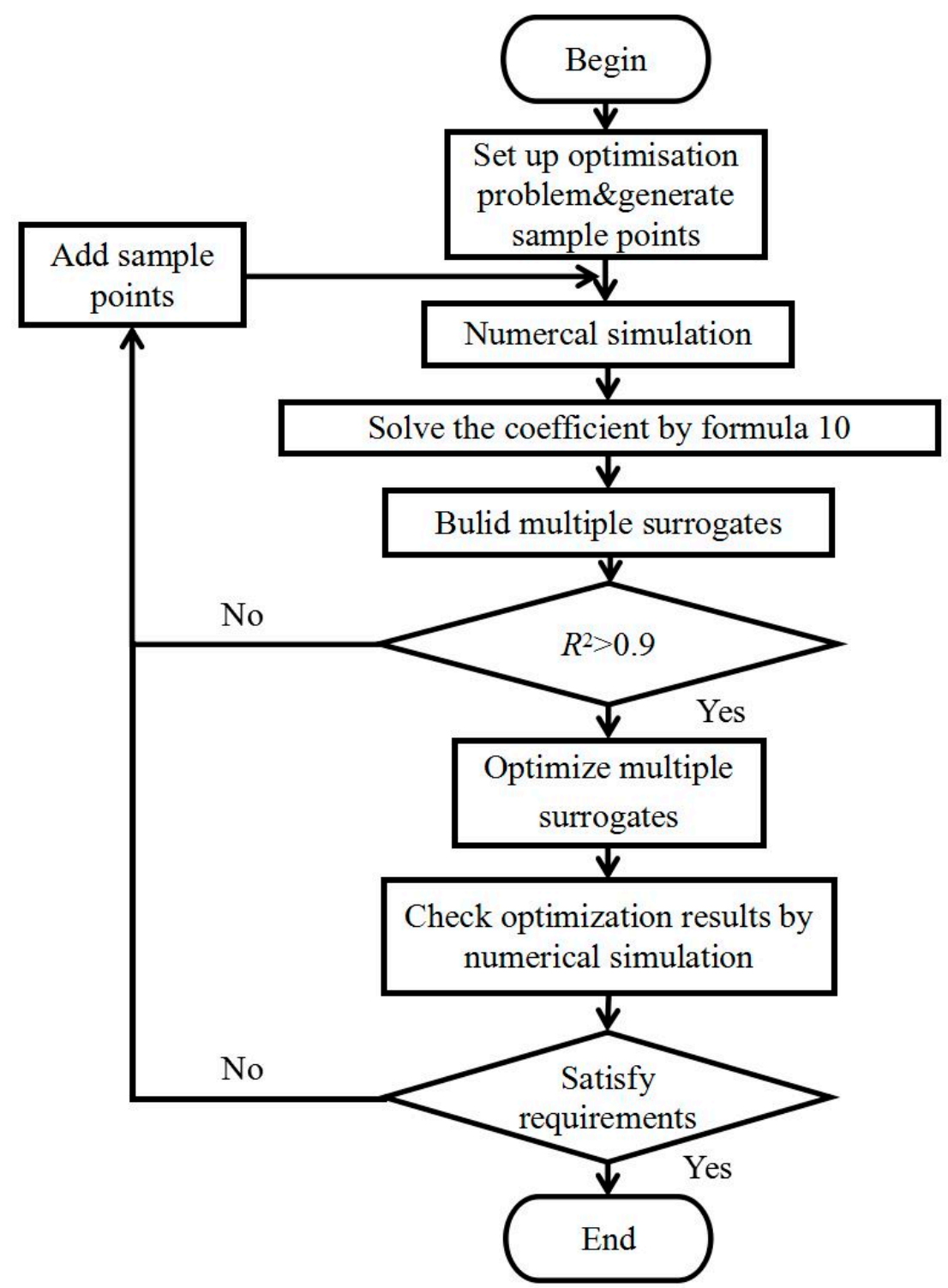

Figure 1. Flow chart of the multiple-surrogate-based optimization.

1. Determine the optimization problem, including objectives, constraints, and design variables, etc., and generate sample points of design variables as training points and testing points with the help of Latin hypercube sampling (LHS) [23]. LHS can more efficiently estimate the overall mean of response than estimation based on random sampling.

2. Perform the numerical simulation at the training points and testing points to obtain the interest responses.

3. Build the surrogates, including PRS, RBFNN, and KRG (reference for the method of construction $[9,20,21])$, respectively. With the minimum GMSE of the test points, solve the coefficients in Equation (10) and construct the multiple surrogates.

4. According to Equation (13), solve the determination coefficient $R^{2}$. Check whether $R^{2}$ is greater than 0.9. If it is greater than 0.9 , go to the next step. Otherwise add the current training points, and return to step 2 to rebuild the multiple surrogates.

5. Optimize the multiple surrogates and check the boundary conditions by numerical simulation. If not, add the current training points, and return to step 2 to rebuild the multiple surrogates. Otherwise, the optimization is completed. 


\section{Design of Segmented Mirror with a GRoC Actuation System}

\subsection{Design Requirements}

The primary mirror of an on-orbit assembled telescope consists of six hexagonal segmented mirrors made of Zerodur (point-to-point distance is $200 \mathrm{~mm}$ and the GRoC is $2406 \mathrm{~mm}$ ). Using the JWST for reference, as shown in Figure 2, there is a GRoC on each segmented mirror's back. The GRoC actuation system comprises an actuator that pushes and pulls against the center of the mirror and six struts to react the force at the six corners of the mirror. The GRoC system can effectively change the GRoC by introducing undesired deformation called GRoC actuation residual.

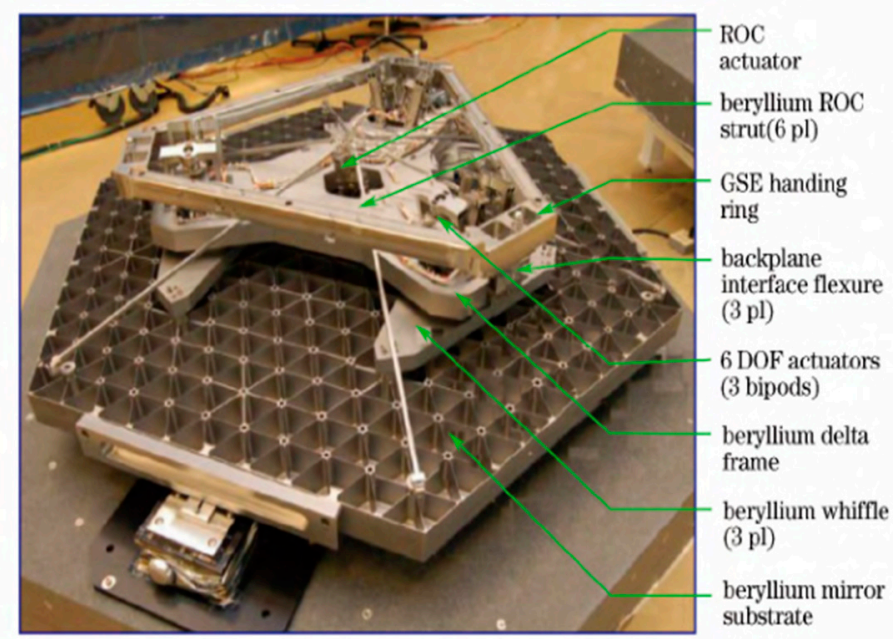

Figure 2. The James Webb Space Telescope (JWST) segmented mirror back [24].

A segmented mirror with a GRoC actuation system was designed in accordance with the process in Figure 3. The specific method of traditional mirror design can be referred to [25], and this work focuses on the support design and the design of the activation point's position.

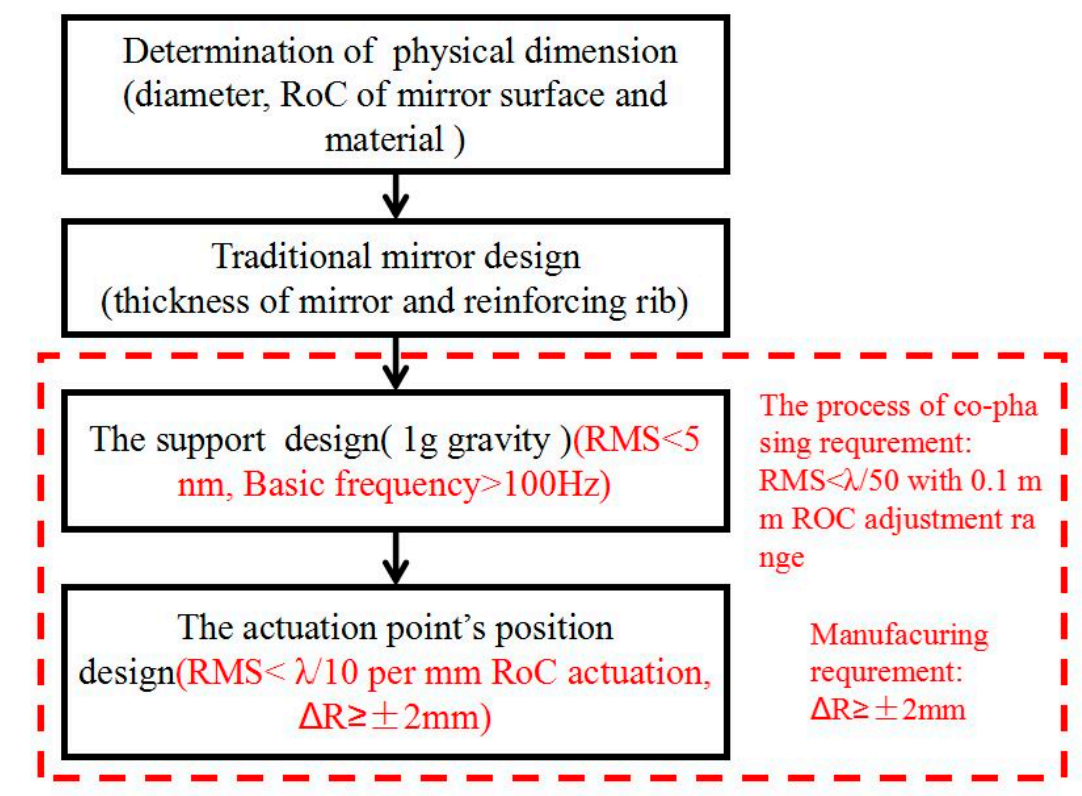

Figure 3. The design process and design requirement of the segmented mirror. 
Space mirrors are always fabricated on the ground and launched into space, and the surface accuracy could be degraded by gravity relief. Therefore, the disturbance of gravity should be considered in the support design. According to the optical requirement of cophase, the segmented mirror's required surface accuracy should not exceed $\lambda / 50$ including accuracy degradation caused by the disturbance of gravity, manufacturing error, and the GRoC actuation residual. The specific design requirements are introduced in Figure 3. In orbit, the cophasing process needs the GRoC actuation to be $\pm 0.1 \mathrm{~mm}$, then the surface shape error RMS of residual should be less than $\lambda / 10$ per millimeter of GRoC actuation $(\lambda=632.8 \mathrm{~nm})$.

\subsection{Support Design of the Segmented Mirror}

\subsubsection{Problem Description}

The mirror is supported by three flexures located on its back. The supporting flexure's structural parameters and the supportive position were optimized under the environment of $1 \mathrm{~g}$ gravity (direction parallel to the mirror surface). The location of the design variables is shown in Figure 4.

$$
\begin{gathered}
\min X_{\mathrm{RMS}}=f_{1}\left(t_{1}, t_{2}, h_{1}, h_{2}, l_{1}, p\right), \\
\text { s.t. }\left\{\begin{array}{c}
1 \mathrm{~mm} \leq t_{1}, t_{2} \leq 5 \mathrm{~mm} \\
1 \mathrm{~mm} \leq h_{1}, h_{2} \leq 7 \mathrm{~mm} \\
20 \mathrm{~mm} \leq l_{1} \leq 90 \mathrm{~mm} \\
5 \mathrm{~mm} \leq p \leq 20 \mathrm{~mm}
\end{array}\right.
\end{gathered}
$$

where $X_{\text {RMS }}$ is surface shape error RMS under a gravity of $1 \mathrm{~g}, h_{1}$ and $h_{2}$ are the height of the upper and lower grooves of the flexure, $t_{1}$ and $t_{2}$ are the width of the upper and lower grooves of the flexure, $p$ is the height of the flexure, and $l_{1}$ is the distance between the flexure support position and the center of the segmented mirror.

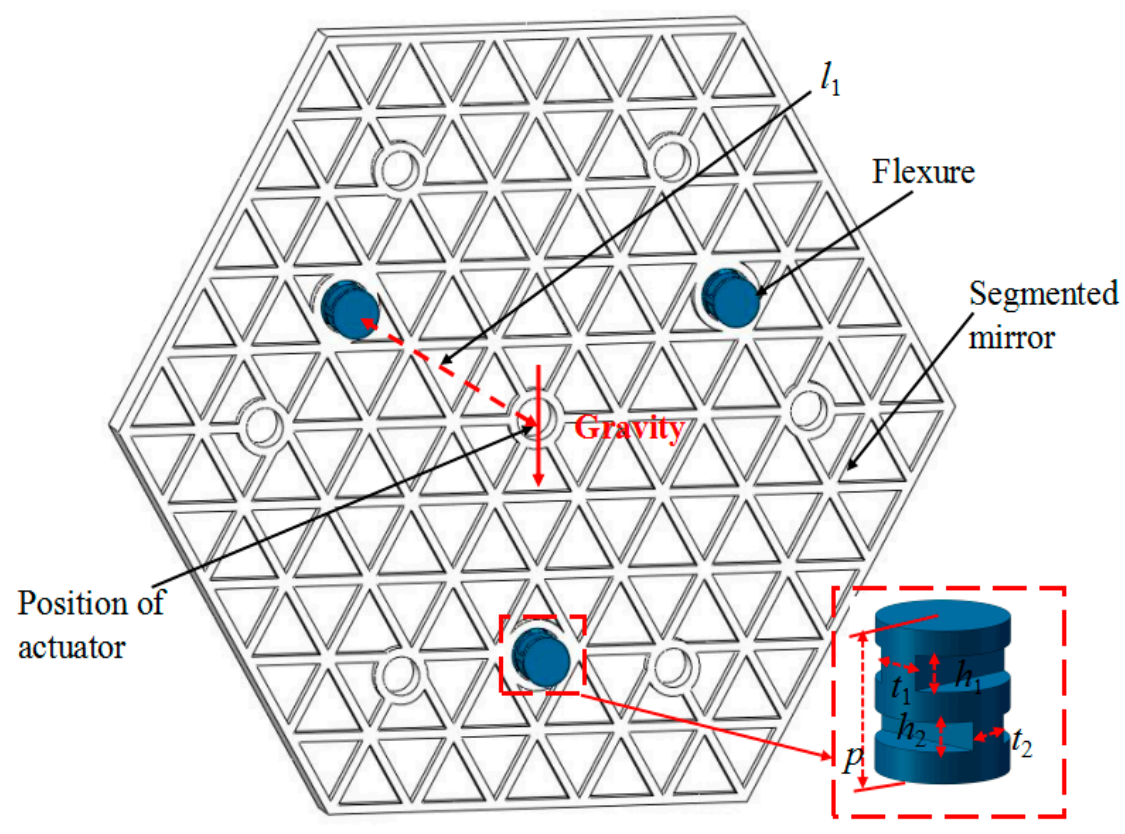

Figure 4. The optimization variables of support design.

\subsubsection{Results of Optimization}

After 125 iterations, the design requirement in Section 3.1 was met by a multi-island genetic algorithm (MIGA) [26] (the population number is 5, the island number is 4 , and the evolution number 
is 10). At that time, the surface shape error RMS was $4.5 \mathrm{~nm}$, and the basic frequency of the segmented mirror and the flexures was $150 \mathrm{~Hz}$. The design variables before and after optimization are shown in Table 1. The displacement map and the surface shape error map of the optimum are shown in Figures 5 and 6 , respectively.

Table 1. Design variables before and after optimization.

\begin{tabular}{ccc}
\hline Variable & Initial Value & Optimization Result \\
\hline$l_{1}$ & $50 \mathrm{~mm}$ & $52.5 \mathrm{~mm}$ \\
$t_{1}$ & $3 \mathrm{~mm}$ & $3.9 \mathrm{~mm}$ \\
$t_{2}$ & $4 \mathrm{~mm}$ & $3.5 \mathrm{~mm}$ \\
$h_{1}$ & $5 \mathrm{~mm}$ & $4.5 \mathrm{~mm}$ \\
$h_{2}$ & $5 \mathrm{~mm}$ & $4.5 \mathrm{~mm}$ \\
$p$ & $15 \mathrm{~mm}$ & $12 \mathrm{~mm}$ \\
\hline
\end{tabular}

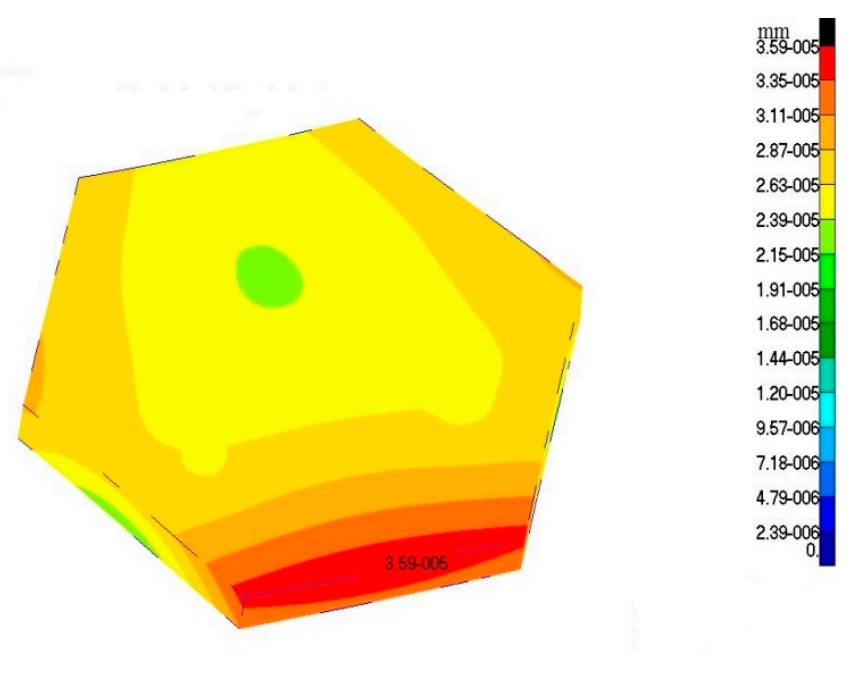

Figure 5. The displacement map of the optimum.

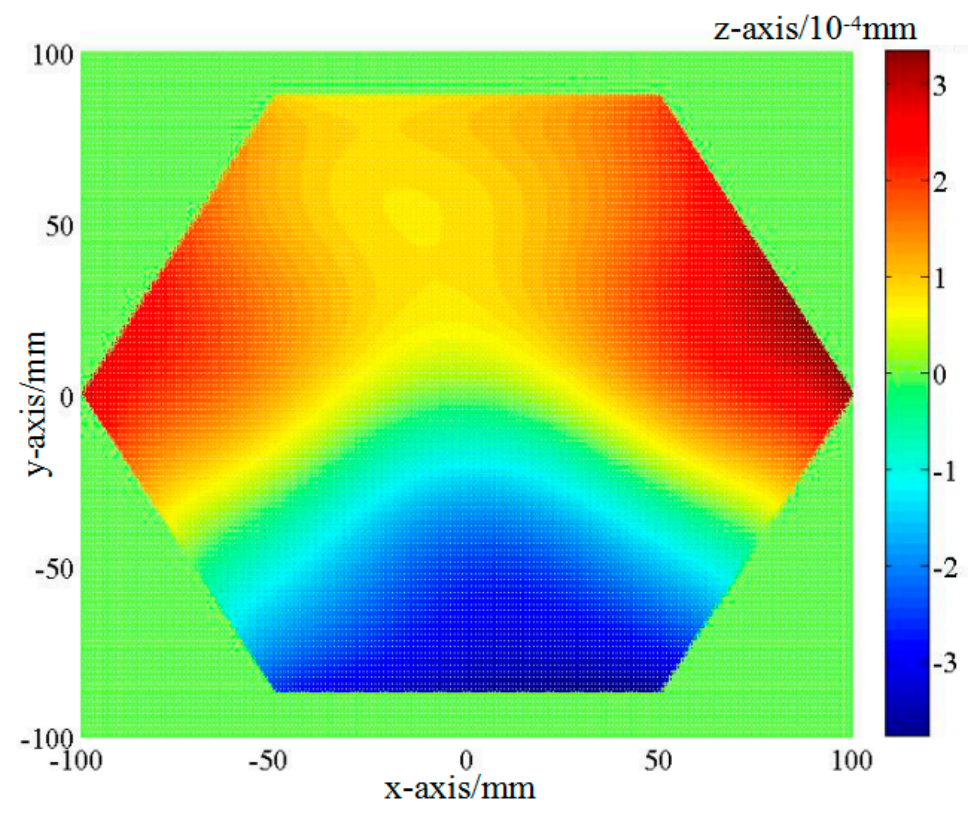

Figure 6. The surface shape error map of the optimum. 


\subsection{Actuation Point's Position Design of Segmented Mirror}

\subsubsection{Problem Description}

After the segmented mirror support design was completed, we optimized the actuation point's position of the GRoC actuation system on the segmented mirror's back with the help of proposed multiple surrogates. Due to the surface shape error RMS of residual, the stress and the amount of GRoC actuation are proportional to the actuator's output. The influence of the actuator on the optimization object can be eliminated by division. In other words, the surface shape error RMS per millimeter of GRoC actuation has nothing to do with the actuator's output, and the same is true of the maximum local stress per millimeter of GRoC actuation. In this optimization, the output of the actuator was set as $1 \mathrm{~N}$. The design variables of the actuation point's position are shown in Figure 7, where $h$ is the depth of the actuation point, $l_{2}$ is the distance between the actuation point of the strut and the actuation point of the actuator, and $d_{1}$ and $d_{2}$ are the diameters of the actuation point of the actuator and the strut, respectively. Due to the fact that the range of GRoC actuation is required to be greater than $2 \mathrm{~mm}$, in order to ensure the normal work of the mirror, the maximum local stress should be checked for strength. The objective functions of the activation point's position are constructed as follows:

$$
\begin{gathered}
\min Y_{\mathrm{RMS}}=f\left(d_{1}, d_{2}, l_{2}, h\right), \\
\text { s.t. }\left\{\begin{array}{c}
0<h<10 \mathrm{~mm} \\
5 \mathrm{~mm} \leq d_{1} \leq 20 \mathrm{~mm} \\
5 \mathrm{~mm} \leq d_{2} \leq 20 \mathrm{~mm} \\
20 \mathrm{~mm} \leq l \leq 90 \mathrm{~mm} \\
2 \delta_{\mathrm{MAX}} \leq \frac{\delta_{s}}{n_{s}}
\end{array},\right.
\end{gathered}
$$

where $Y_{\text {RMS }}$ and $\delta_{\text {MAX }}$ are the surface shape error RMS of residual and the maximum local stress per millimeter of GRoC actuation, the tensile yield strength of Zerodur, $\delta_{s}$, is $57 \mathrm{MPa}$, and the safety factor, $n_{s}$, is 2 .

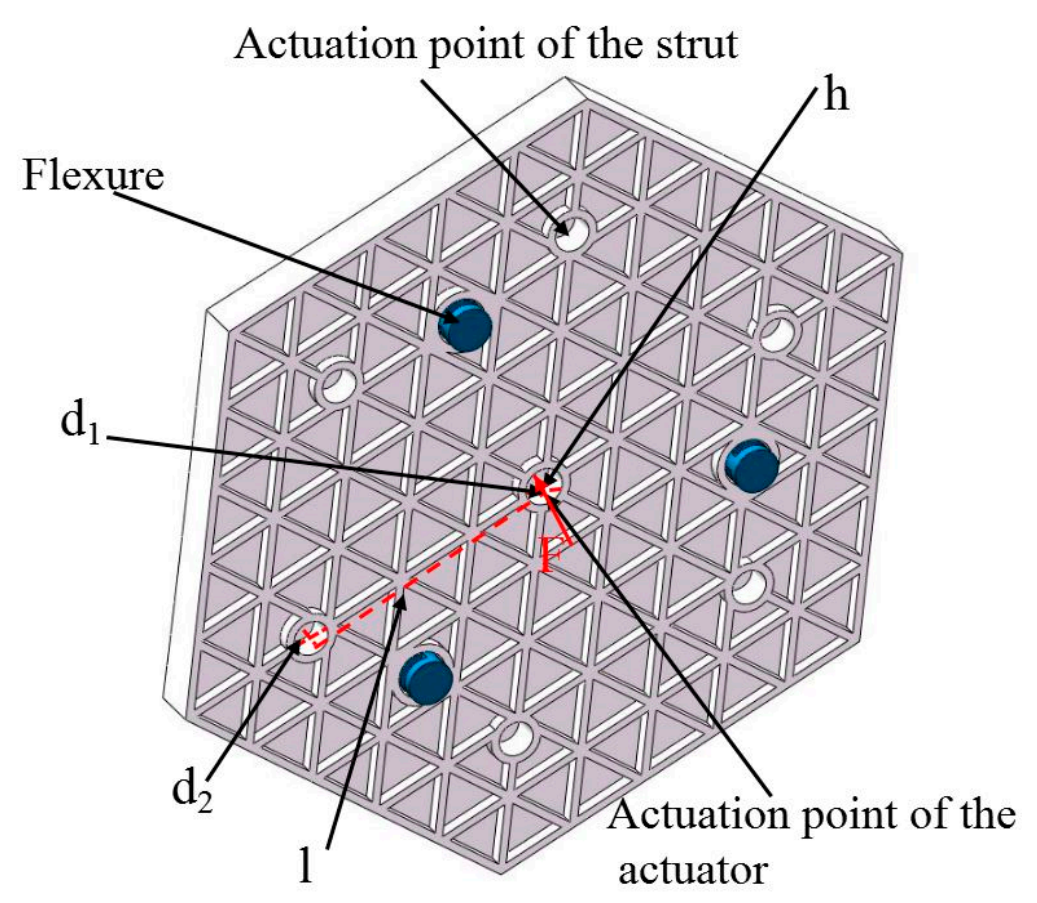

Figure 7. The optimization variables of the actuation point's position design. 


\subsubsection{Procedure of the Actuation Point's Position Optimization}

With the help of Figure 8, the procedure of the actuation point's position optimization is outlined in the following steps.

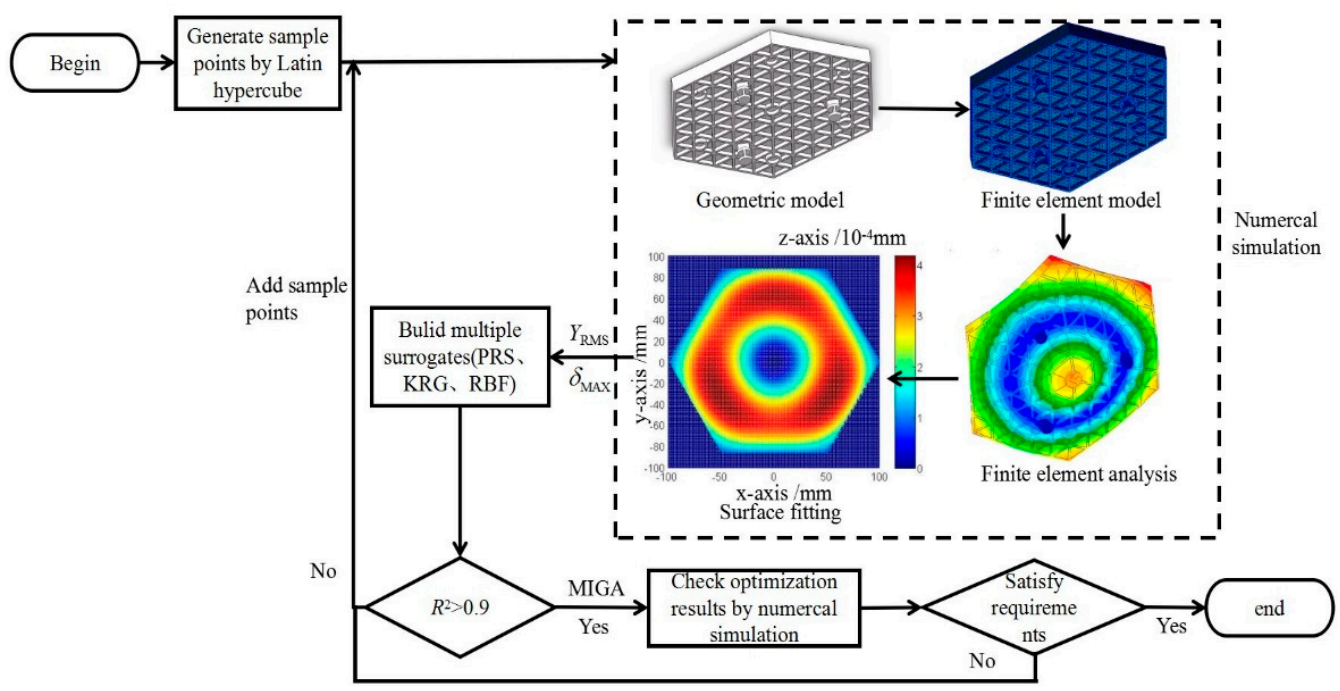

Figure 8. Framework of the actuation point's position optimization.

1. Generate 20 training points and 10 testing points of design variables by LHS.

2. Take the sampled points into the numerical simulation. In the numerical simulation, a geometric model is built at a sampled point by computer-aided design (CAD) software, Solidworks. Then analyze the geometric model to get figure deformation and the maximum local stress of the mirror with the finite element analysis (FEA) software Patran and Nastran. Finally, the surface shape error RMS and the amount of GRoC actuation are evaluated by using MATLAB.

3. Divide the surface shape error RMS and the maximum local stress by the amount of GRoC actuation to get $Y_{\text {RMS }}$ and $\delta_{\text {MAX }}$. Build RBFNN, PRS, and KRG, respectively, using $Y_{\text {RMS }}, \delta_{\text {MAX }}$, and training points, then construct multiple surrogates according to Section 2.1.

4. Solve the determination coefficient $R^{2}$ of multiple surrogates and check whether it is greater than 0.9. If not, add 5 training points and return to step 2.

5. Search the optimum of the multiple surrogates through MIGA (population number is 5, island number is 4 , evolution number is 10 ).

6. Verify whether the optimum meets boundary conditions. If not, add 5 training points and return to step 2.

\subsubsection{Results of Optimization}

After 156 iterations of MIGA, the optimum was obtained. At that time, the number of training points was $55, Y_{\mathrm{RMS}}$ was $0.09 \lambda$, and $\delta_{\mathrm{MAX}}$ was $1.51 \mathrm{MPa}$, which met the design requirements in Section 3.1. The displacement map and the surface shape error map of the optimal design are shown in Figures 9 and 10, respectively. The changes in design variables before and after the optimization are shown in Table 2 and the coefficients of each reconstruction of multiple surrogates are shown in Table 3. 

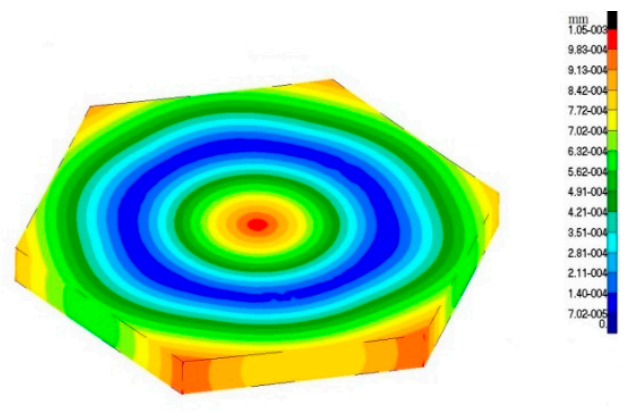

Figure 9. The displacement map of the optimum.

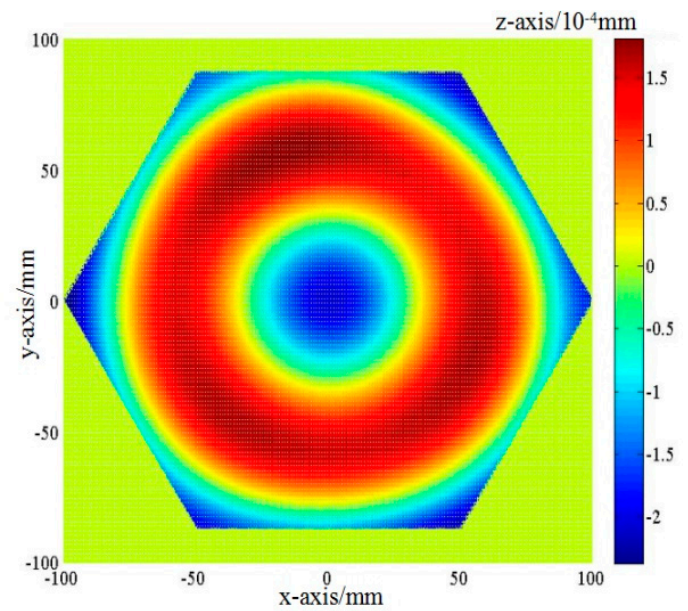

Figure 10. The surface shape error map of the optimum.

Table 2. Design variables and optimization results.

\begin{tabular}{ccc}
\hline Variable & Initial Value & Optimization Result \\
\hline $\mathrm{h}$ & $5 \mathrm{~mm}$ & $7.5 \mathrm{~mm}$ \\
$\mathrm{~d} 1$ & 15 & $9.4 \mathrm{~mm}$ \\
$\mathrm{~d} 2$ & 10 & $8.7 \mathrm{~mm}$ \\
$\mathrm{l} 2$ & 80 & $65 \mathrm{~mm}$ \\
\hline
\end{tabular}

Table 3. The coefficients of multiple surrogates.

\begin{tabular}{ccccccc}
\hline Output Response & \multicolumn{3}{c}{$\Upsilon_{\text {RMS }}$} & & & $\delta_{\text {MAX }}$ \\
\hline Surrogate & RBF & PRS & KRG & RBF & PRS & KRG \\
\hline 20 & 0 & 0 & 1 & 1 & 0 & 0 \\
25 & 0 & 0 & 1 & 1 & 0 & 0 \\
30 & 0.25 & 0.55 & 0.20 & 1 & 0 & 0 \\
35 & 0 & 1 & 0 & 0.82 & 0.18 & 0 \\
40 & 0 & 0.29 & 0.71 & 0.78 & 0.22 & 0 \\
45 & 1 & 0 & 0 & 0.69 & 0.31 & 0 \\
50 & 0.69 & 0.31 & 0 & 0.65 & 0.35 & 0 \\
55 & 0.47 & 0 & 0.53 & 0.33 & 0.33 & 0.24 \\
\hline
\end{tabular}

\subsubsection{Approximation Ability Analysis}

A comparison of determination coefficient $R^{2}$ between the multiple surrogates and the surrogates (RBFNN, PRS, and KRG) is shown in Figure 11 to verify the approximate ability of the multiple surrogates. It can be seen that the $R^{2}$ of the multiple surrogates is always the largest each time the surrogates are rebuilt. When the multiple surrogates met the decision condition $\left(R^{2}>0.9\right)$ with 55 sampling points, the coefficient of determination is shown in Table 4. 


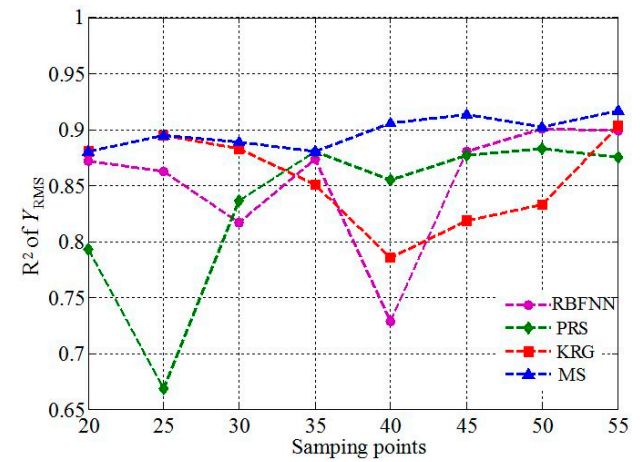

(a)

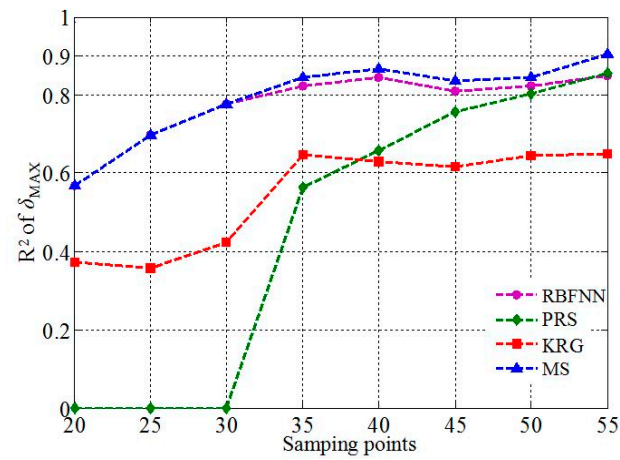

(b)

Figure 11. Determination coefficient of multiple surrogates: (a) $Y_{\text {RMS }}$ and (b) $\delta_{\text {MAX }}$.

Table 4. The determination coefficient of surrogates.

\begin{tabular}{ccccc}
\hline Surrogate & MS & PRS & RBFNN & KRG \\
\hline$Y_{\text {RMS }}$ & 0.92 & 0.87 & 0.90 & 0.90 \\
$\delta_{\text {MAX }}$ & 0.91 & 0.85 & 0.85 & 0.64 \\
\hline
\end{tabular}

\subsubsection{Computational Efficiency Analysis}

Since the computational cost of the solving coefficient and the iteration of the MIGA were negligible compared with the numerical simulation running, the number of iterations of the numerical simulation can be used to characterize the computational efficiency in this work. The comparison between the direct optimization by numerical simulation and the multiple-surrogate-based optimization is shown in Figure 12. Both optimizations used the same MIGA (the population number is 5, the number of islands is 4 , and the number of evolution is 10 ).

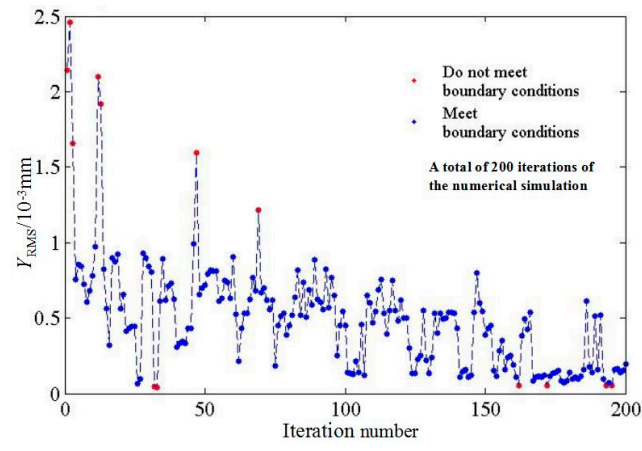

(a)

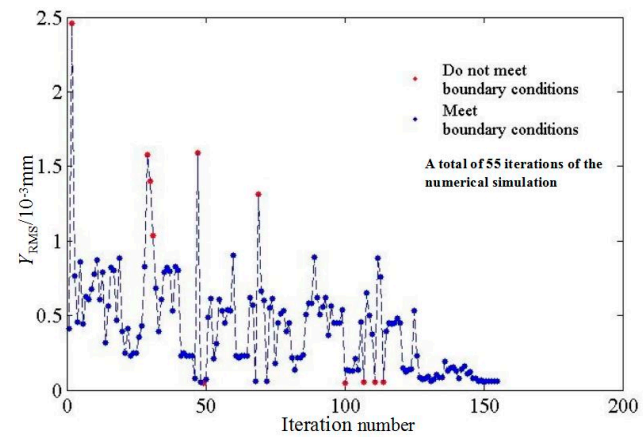

(b)

Figure 12. Iterative process of optimization: (a) the optimization by numerical simulation and (b) the multiple-surrogate-based optimization.

It can be seen that after 200 iterations of the direct optimization by the numerical simulation, the convergence condition was still not satisfied in Figure 12a. In comparison, the multiple-surrogate-based optimization had been iterated 158 times to meet the convergence condition and the design requirement (a total of 55 iterations of the numerical simulation) in Figure 12b.

\section{Discussion}

In this paper, a segmented mirror with a GRoC actuation system was designed. To solve the problem of insufficient approximation ability of existing surrogates, we proposed multiple surrogates to optimize the actuation point's position on the segmented mirror's back. 
The comparison of approximation ability between the multiple surrogates and some existing surrogates (PRS, RBFNN, and KRG) in Figure 11 shows that the multiple surrogates' determination coefficient remained the highest during the iteration of the algorithm. It was the first to reach the decision condition $\left(R^{2}>0.9\right)$ with 55 sampling points, and the determination coefficients of all surrogates at that time are shown in Table 4. The comparison of computational efficiency is shown in Figure 12, and it can be seen that the multiple surrogates needed 55 iterations of the numerical simulation to satisfy the convergence condition. In comparison, the direct optimization cannot fully converge after 200 iterations of numerical simulation. This might be because the numerical simulation is too complicated, and the optimum is affected by the local minima. The approximation of the numerical simulation is equivalent to smooth filtering, which reduces the local minima's influence and makes the optimization easier to converge. Some conclusions can be drawn:

1. The optimization methodology used in this work provides a solution for designing a segmented mirror with a GRoC actuation system.

2. The proposed multiple surrogates have higher approximation ability than a single surrogate (PRS, RBFNN, and KRG), and at the same time, solve the problem of difficulty in choosing a reasonable surrogate in an approximation optimization.

3. The proposed multiple-surrogate-based optimization methodology effectively reduces the computational cost of optimization problems and makes the optimization problems easier to converge. In addition, this methodology is also suitable to be extended to other complex optimization problems.

It should be noted that although our multiple surrogates achieved good results in this work, other surrogates such as deep learning could also be added to them. Hence, more surrogates will be added to our multiple-surrogate-based optimization methodology in the future.

Author Contributions: Project administration, S.W.; methodology, J.D. and S.W.; software, Z.L.; writing-original draft, S.W.; writing-review and editing, S.X. and B.X. All authors have read and agreed to the published version of the manuscript.

Funding: This research was funded by the National key research and development plan of China, grant number 2016YFE020500 and the National Natural Science Foundation of China (NSFC), grant number 61905241.

Conflicts of Interest: The authors declare no conflict of interest.

\section{References}

1. Jiang, Y.H.; Chen, X.L.; Wu, Y.J.; Li, Y. Error analysis of curvature radius of segmented mirror. Spacecr. Recovery Remote Sens. 2017, 38, 102-108.

2. Annu, J.; Padmakar, P.; Melvin, K.J. Creating a large aspheric primary mirror using spherical segments. Exp. Astron. 2020. [CrossRef]

3. Nijenhuis, J.; Hamelinck, R.; Braam, B. The opto-mechanical performance prediction of thin mirror segment for the E-ELT. In Modern Technologies in Space-and Ground-Based Telescopes and Instrumentation II; International Society for Optics and Photonics: Bellingham, DC, USA, 2012; Volume 7733, p. 7732H.

4. David, M.; James, B.; Jake, L. Cryogenic radius of curvature matching for JWST primary mirror segments. In Astronomical and Space Optical Systems; International Society for Optics and Photonics: Bellingham, DC, USA, 2009; Volume 7439, p. 743916.

5. James, B.H.; David, M.C.; Larkin, B.C. The optical metrology system for cryogenic testing of the JWST primary mirror segments. In Optical Manufacturing and Testing IX; International Society for Optics and Photonics: Bellingham, DC, USA, 2011; Volume 8126, p. 81260P.

6. Adam, R.C.; Acton, D.S.; Paul, D.A.; Allison, A.B.; Paul, A.L.; Duncan, M.S. Aligning and maintaining the optics for the James Webb Space Telescope(JWST) on-orbit: The wavefront sensing and control concept of operations. In Space Telescopes and Instrumentation I: Optical, Infrared, and Millimeter; International Society for Optics and Photonics: Bellingham, DC, USA, 2006; Volume 6265, p. 62650X.

7. Wang, Z.S.; Zhang, J.X.; Wang, J.X.; He, X.; Fu, L.L.; Tian, F.X.; Liu, X.F.; Zhao, Y.C. A back propagation network based optimizing model of space-based large mirror structure. Optik 2019, 179, 780-786. [CrossRef] 
8. Hong, Z.; Yu, S.; Chenchen, L.; Haoran, W. Optimal design of the sealing structure of a hydraulic cylinder on the basis of a surrogated model. Adv. Mater. Sci. Eng. 2020, 2020, 1753964.

9. Xiang, X.S.; Tian, Y.; Xiao, J.H.; Zhang, X.Y. A clustering-based surrogate-assisted multi-objective evolutionary algorithm for shelter location problem under uncertainty of road networks. IEEE Trans. Ind. Informat. 2020, 16, 7544-7555. [CrossRef]

10. Ribeiro, L.G.; Maia, M.A.; Parente, E.; Cartaxo de melo, A.M. Surrogate based optimization of functionally graded plates using radial basis functions. Compos. Struct. 2020, 252, 112677. [CrossRef]

11. Wang, P.H.; Chen, Z.G.; Feng, Y.K. Many-objective optimization for a deep-sea aquaculture vessel based on improved RBF neural network surrogate model. J. Mar. Sci. Technol. 2020, 1-24. [CrossRef]

12. Hsiao, J.C.; Kumar, S.; Chou, C.L.; Kam, T.Y. Shape design optimization of a robot arm using a surrogate-based evolutionary approach. Appl. Sci. 2020, 10, 2223. [CrossRef]

13. Ahmed, S.; Grabher, C.; Kim, H.J.; Koseki, T. Multifidelity surrogate assisted rapid design of transverse-flux permanent magnet linear synchronous motor. IEEE Trans. Ind. Electron. 2020, 67, 7280-7289. [CrossRef]

14. Rafiee, V.; Faiz, J. Robust design of an outer rotor permanent magnet motor through six-sigma methodology using response surface surrogate model. IEEE Trans. Magn. 2019, 55, 8107110. [CrossRef]

15. Chau, N.L.; Le, H.G.; Dao, T.P.; Dang, V.A. Design and optimization for a new compliant planar spring of upper limb assistive device using hybrid approach of RSM-FEM and MOGA. Arab. J. Sci. Eng. 2019, 44, 7441-7456. [CrossRef]

16. Hakjin, L.; Yeongmin, J.; Duck-Joo, L.; Seongim, C. Surrogate model based design optimization of multiple wing sails considering flow interaction effect. Ocean Eng. 2016, 121, 422-436.

17. Zhenxu, S.; Ye, Z.; Guowei, Y. Surrogate based optimization of aerodynamic noise for streamlined shape of high speed trains. Appl. Sci. 2017, 7, 196.

18. Guo, Q.; Hang, J.T.; Wang, S.A.; Hui, W.Z.; Xie, Z.H. Buckling optimization of variable stiffness composite cylinders by using multi-fidelity surrogate models. Thin Walled Struct. 2020, 156, 107014. [CrossRef]

19. Das, S.; Tesfamariam, S.; Chen, Y.Y.; Qian, Z.C.; Tan, P.; Zhou, F.L. Reliability-based optimization of nonlinear energy sink with negative stiffness and sliding friction. J. Sound Vib. 2020, 485, 115560. [CrossRef]

20. Deng, S.Y.; Yao, J.Y.; Wang, L.L.; Xin, J.Q.; Hu, N. Comparative studies of surrogate models for response analysis of mistuned bladed disks. Int. J. Comput. Methods 2020, 17, 2050012. [CrossRef]

21. Gao, H.F.; Zio, E.; Wang, A.E.; Bai, G.C.; Fei, C.W. Probabilistic-based combined high and low cycle fatigue assessment for turbine blades using a substructure-based kriging surrogate model. Aerospace Sci. Technol. 2020, 104, 105957. [CrossRef]

22. Viana, F.A.C.; Haftka, R.T. Cross validation can estimate how well prediction variance correlates with error. AIAA J. 2009, 47, 2266-2270. [CrossRef]

23. Zhang, F.; Cheng, L.; Wu, M.Y.; Xu, X.Y.; Wang, P.C.; Liu, Z.B. Performance analysis of two-stage thermoelectric generator model based on Latin hypercube sampling. Energy Convers. Manag. 2020, 221, 113159. [CrossRef]

24. Mark, C. Status of the James Webb space telescope (JWST). In Space Telescopes and Instrumentation 2008: Optical, Infrared, and Millimeter; International Society for Optics and Photonics: Bellingham, DC, USA, 2008; Volume 7010, p. 70100L.

25. Pierre, Y.B. The Design and Construction of Large Optical Telescopes; Springer: Berlin, Germany, 2003; pp. $146-155$.

26. Liang, T.; Lu, H. A novel method based on Multi-Island Genetic Algorithm improved variational mode decomposition and multi-features for fault diagnosis of rolling bearing. Entropy 2020, 22, 995. [CrossRef]

Publisher's Note: MDPI stays neutral with regard to jurisdictional claims in published maps and institutional affiliations.

(C) 2020 by the authors. Licensee MDPI, Basel, Switzerland. This article is an open access article distributed under the terms and conditions of the Creative Commons Attribution (CC BY) license (http://creativecommons.org/licenses/by/4.0/). 